\title{
P. Seele Polymorphic Eruption of Pregnancy (PEP) und ihre H.-D. Göring Abgrenzung zu anderen Schwangerschaftsdermatosen
}

Two Cases of Polymorphic Eruption of Pregnancy (PEP) - Differentiation to Other Dermatosis of Pregnancy

\section{Zusammenfassung}

Es wird über eine 29-jährige und eine 27-jährige Primigravida berichtet, die zu Beginn des sechsten Schwangerschaftsmonates ausgedehnte stark juckende Hautveränderungen entwickelten. Bei einer der Frauen war primär die abdominelle Region befallen, bei der anderen die Extremitäten. Durch klinische, histologische und immunologische Untersuchungen konnte in beiden Fällen die Diagnose polymorphic eruption of pregnancy (PEP) gestellt und das Vorliegen anderer Schwangerschaftsdermatosen ausgeschlossen werden. In einem Fall lagen ein Pemphigus-like-Antikörper und ein erhöhter Titer gegen Masernvirus vor, die ohne pathogenetische Bedeutung waren. Aufgrund des ausgeprägten Juckreizes und Leidensdruckes erhielten beide Patientinnen intern niedrig dosiert Steroide, darunter kam es zum Sistieren des Pruritus und zur Abheilung der Hautveränderungen. Anhand des Zeitpunktes ihres Auftretens in der Schwangerschaft, des klinischen Bildes, der histologischen und immunologischen Befunde wird eine differenzialdiagnostische Abgrenzung zu den anderen Schwangerschaftsdermatosen Pruritus gravidarum, Prurigo gestationis, Herpes gestationis, Papulöse Dermatitis in der Schwangerschaft, Autoimmun-Progesteron-Dermatitis, pruritische Follikulitis und Erythema nodosum gravidarum vorgenommen.

\section{Abstract}

We report on a 29- and a 27-year-old primigravida, which both developed pruritic eruptions in the end of the 6th month of pregnancy. The lesions began in one case on the abdomen and in the other case on the arms and legs. By means of our clinical, histological and immunological investigations we could confirm the diagnosis polymorphic eruptions of pregnancy (PEP) and exclude the other diseases of pregnancy. In one case we found a pemphigus-like-antibody and an elevated titer against the measles-virus, both without pathogenetic meaning. Because of the extreme pruritus and the impairment of quality of life, we gave both patients a therapy with oral corticosteroids with good effect. We excluded other dermatosis of pregnancy because of the typical beginning and the clinical, immunological and histological results.

\section{Einleitung}

Dermatosen in der Schwangerschaft stellen eine Herausforderung dar. Sie können in dieser besonderen Situation ein diagnostisches und therapeutisches Problem darstellen und die Schwan- gere sowie ihre Angehörigen und den behandelnden Gynäkologen beunruhigen. Wegen der befürchteten Schäden für das Kind wird häufig auf eine Behandlung gedrängt. Diese wiederum ist je nach vorliegender Schwangerschaftsdermatose sehr differenziert. Kenntnisse der Dermatologen über Schwangerschaftsder\section{Hautklinik und Immunologisches Zentrum des Städtischen Klinikums Dessau (Chefarzt: Prof. Dr. med. H.-D. Göring) \\ Herrn Prof. Dr. med. B. Wüthrich, Zürich, zum 65. Geburtstag gewidmet}


matosen sind daher unverzichtbar. Das betrifft auch das Krankheitsbild der polymorphic eruption of pregnancy (PEP), das eher zu den häufigeren Schwangerschaftsdermatosen gehört. Trotzdem finden sich im deutschen dermatologischen Schrifttum der letzten 10 Jahre nur ganz vereinzelte Erwähnungen dieser Dermatose. Wir stellen im Folgenden 2 Patientinnen mit PEP vor.

\section{Kasuistik 1}

\section{Anamnese}

Die 27jährige kroatische Patientin stellte sich im sechsten Monat ihrer ersten Schwangerschaft in unserer Klinik vor. Ungefähr zwei Wochen vorher hätten sich bei der bis dahin hautgesunden Patientin, am Abdomen beginnend, sehr stark juckende Papeln und Plaques entwickelt. Diese breiteten sich im Verlauf auf die Oberschenkel, Arme und den Rücken aus. Außer Folicombin und Jodid wurden keine Medikamente eingenommen.

Dermatologischer Befund: An Armen, Beinen, Abdomen und Rücken finden sich neben Erythemen und Papeln infiltrierte Plaques und oberflächliche Kratzeffekte (Abb. 1). Schleimhäute, Gesicht, Palmae und Plantae frei von Veränderungen.

\section{Diagnostik}

Histologie (läsionale Haut):

Epidermis: unregelmäßige Akanthose, vereinzelt Parakeratose des Stratum corneum, herdförmige Spongiose; Dermis: diskrete perivaskuläre Infiltrate aus Lymphozyten und Histiozyten, mit der Diagnose PEP vereinbar (Abb. 2u.3).

Immunologische Befunde:

DIF (läsionale Haut): keine Fluoreszenzphänomene nachweisbar.

IIF: keine zirkulierenden Antikörper gegen Basalmembran und Interzellularsubstanz nachweisbar (Substrat: Affenösophagus) ANF: nicht nachweisbar (Substrat: HEp2-Zellen).

\section{Therapie und Verlauf}

Externe Behandlung mit 5\% Thesit in Lotio zinci oxidati SR sowie mit Dexamethason Creme LAW, intern Loratadin. Nach vier Tagen klangen darunter Pruritus und Hauterscheinungen deutlich $\mathrm{ab}$, es wurde daher auf eine indifferente hautpflegende externe Therapie gewechselt. Daraufhin Rezidiv an beiden Unterarmen. Wegen des ausgeprägten Leidensdruckes Gabe von Prednisolon $20 \mathrm{mg} /$ die, ausschleichend auf $5 \mathrm{mg} /$ die. Dadurch vollständige Abheilung.

\section{Kasuistik 2}

\section{Anamnese}

29-jährige Phillipina im sechsten Monat ihrer ersten, bis dahin unauffällig verlaufenen Schwangerschaft. Einige Tage vor Vorstellung in unserer Klinik Auftreten von juckenden Hautveränderungen an Armen und Beinen. Rasche Ausbreitung auf Rumpf und Gesäß mit Neigung zur Konfluenz. Medikamente wurden nicht eingenommen.

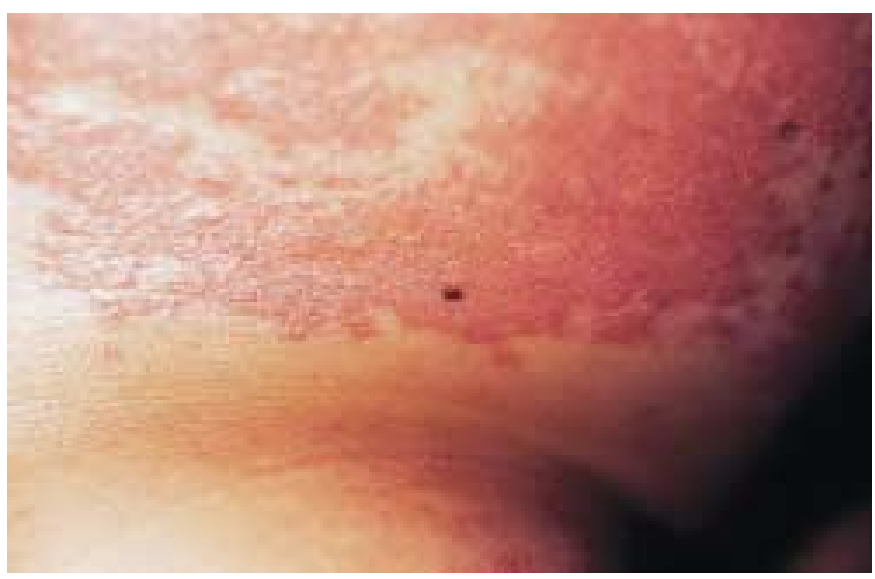

Abb. 1 Papeln und Plaques (Abdomen) - Patientin 1.

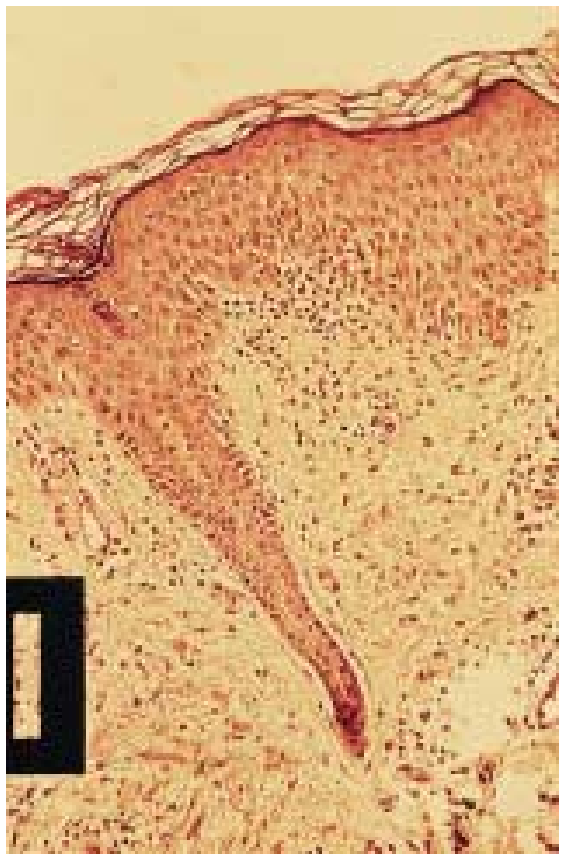

Abb. 2 Histologie PEP (HE 100fach): Epidermis: unregelmäßige Akanthose, herdförmige Spongiose; Dermis: diskrete perivaskuläre Infiltrate aus Lymphozyten und Histiozyten - Patientin 1.

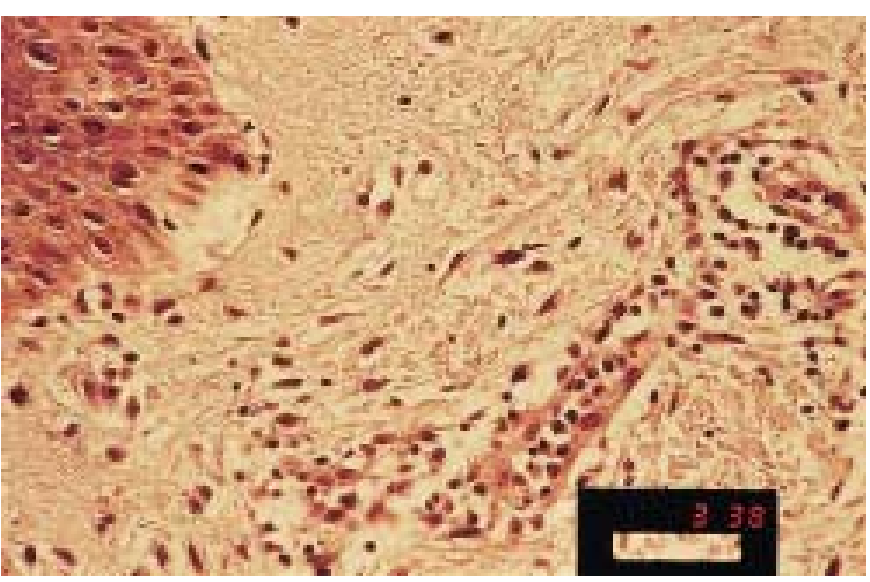

Abb. 3 Histologie PEP (HE 200fach) - Vergrößerung von Abb. 2 (Patientin 1). 
Dermatologischer Befund: An Abdomen, Dekolletee, Mammae und Streckseiten von Armen und Beinen erbs- bis handtellergroße, z. T. konfluierende Erytheme, Papeln und Plaques (Abb. 4 u. 5). Palmae, Plantae, Gesicht und Schleimhäute frei von Veränderungen.

\section{Diagnostik}

Histologie (läsionale Haut):

Epidermis: unregelmäßige Akanthose, herdförmige Spongiose und fleckförmige Exozytose von Lymphozyten, Vakuolisierung und Hyperpigmentation basalnaher Keratinozyten. In der Dermis perivaskuläre Infiltrate im Bereich des oberflächlichen und tiefen Plexus, mit der Diagnose PEP vereinbar.

Immunologische Befunde:

DIF (läsionale Haut): keine Fluoreszenzphänomene nachweisbar.

IIF: keine zirkulierenden Antikörper gegen Basalmembran (Substrat: Affenösophagus), Retikulin und IgA-Endomysium (Substrat: Affendarm) nachweisbar.

Gliadin-Antikörper (ELISA): nicht nachweisbar.

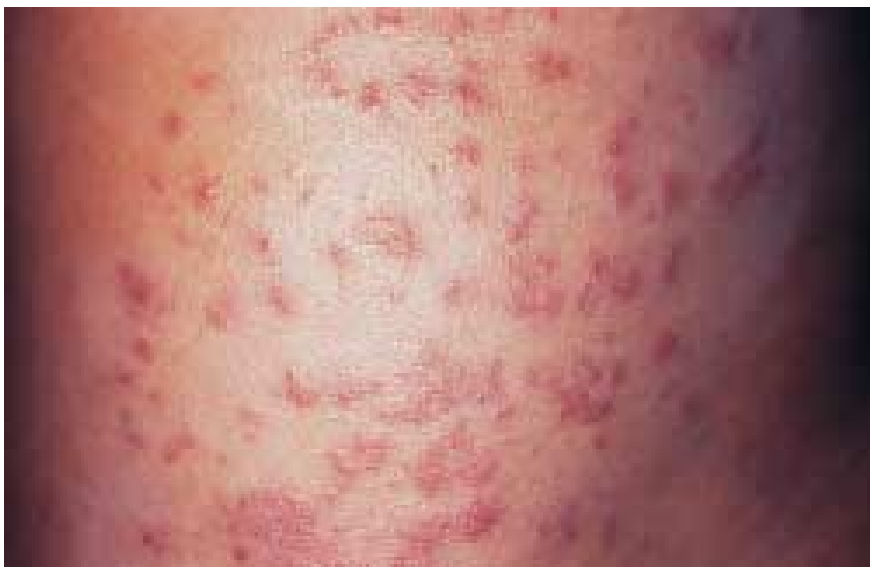

Abb. 4 Papeln und Plaques (Oberschenkel) - Patientin 2.

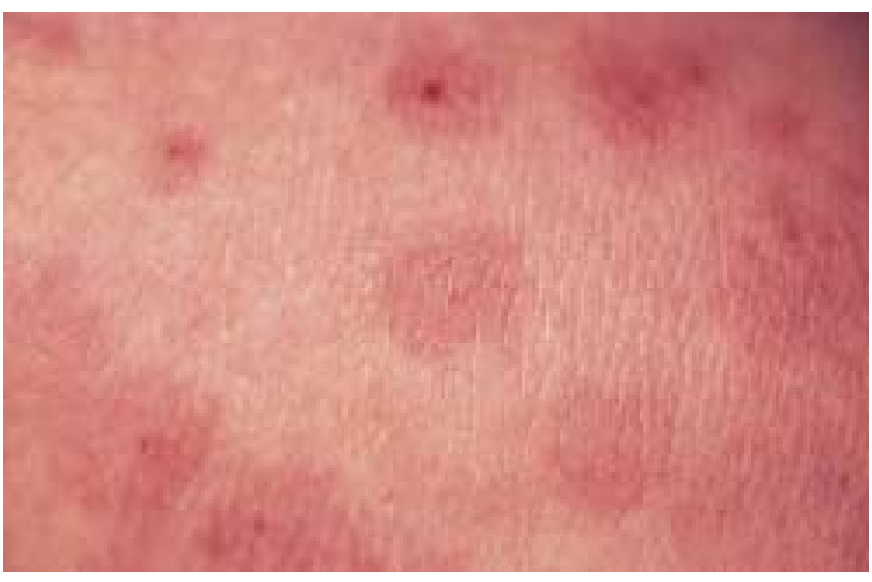

Abb. 5 Erytheme und Exkorationen (Rücken) - Patientin 2.
Antikörper gegen Interzellularsubstanz (ICS): 1:640 (Substrat: Affenösophagus).

IIF nach Absorbtion mit AB-Blutgruppenantigenen: keine zirkulierenden IgG- und IgA-AK gegen ICS nachweisbar (Substrat: $\mathrm{NaCl}$-separierte humane Spalthaut und Affenösophagus).

ANF: $1: 320$ (trivalent, gesprenkelt, Substrat: HEp2-Zellen), ENAScreen: negativ (Substrat: HEp2-Zellen), ds-DNS-AK (Substrat: Crithidia luciliae): negativ.

Klinische Chemie:

BSG 80/88 mm, CRP 15,6 mg/l - sonst Routinelaborparameter unauffällig.

Urinbefund: 10 - 15 Leuko und 6-9 Ery pro Gesichtsfeld, reichlich Plattenepithelien, vereinzelt Bakterien.

Infektionsdiagnostik:

Masern-IgM-AK mit 1,9 positiv ( $\mathrm{NW}<1,1$ ), Masern-IgG-AK mit 3,3 positiv, HSV-1/2-IgG-AK positiv, HSV-IgM-AK negativ, Hepatitis-, Mumps- und Coxsackie-Virus-Serologie negativ.

Allergologische Diagnostik:

Ges-IgE mit 43,2 kU/l normwertig, spezif. IgE - Aeroallergene: negativ.

\section{Therapie und Verlauf}

Stationäre Aufnahme für wenige Tage und externe Therapie mit $5 \%$ Ichthyol in Lotio zinci oxidati SR. Intern wurden zusätzlich $20 \mathrm{mg}$ Prednisolon/die gegeben, später Reduktion auf $10 \mathrm{mg} / \mathrm{die}$. Bei ambulanter Wiedervorstellung nach einer Woche vollständige Abheilung der Hauterscheinungen.

\section{Diskussion}

Spangler et al. beschrieben 1978 Fälle einer Schwangerschaftsdermatose, die sie „pruritic papules of pregnancy (PPP)“ nannten (in [12]). Lawley et al. schlugen 1979 für diese, auch von ihnen vornehmlich im letzten Trimenon der Schwangerschaft beobachtete, Dermatose den Namen „pruritic urticarial papules and plaques of pregnancy (PUPPP)“ vor [10]. Seitdem ist in der englischsprachigen Literatur eine Reihe von Arbeiten über die PUPPP veröffentlicht worden $[3,5-9,14-18]$, z.T. auch unter anderen Bezeichnungen wie „polymorphic eruption of pregnancy (PEP)“, „late onset prurigo of pregnancy“, „toxic erythema of pregnancy“ und „toxaemic rash of pregnancy“. Die Bezeichnung PUPPP wird vorwiegend in den USA verwendet, PEP wird dagegen im europäischen Sprachraum favorisiert. Beide Termini beschreiben allerdings ein und dasselbe Krankheitsbild [2].

Aronson et al. [3] unterscheiden 3 klinische Formen der PEP:

Typ I: hauptsächlich urtikarielle Papeln und Plaques, Typ II: Erytheme, nichturtikarielle Papeln und Blasen, Typ III: Kombination Typ I und II. 
Tab. 1 Schwangerschaftsdermatosen

\begin{tabular}{|c|c|c|c|c|}
\hline Dermatose & Beginn & Morphe & Besonderheiten & Therapie \\
\hline 1. PEP & $\begin{array}{l}\text { (2.) - 3. Trimenon, } \\
\text { selten postpartal }\end{array}$ & $\begin{array}{l}\text { urtikarielle Papeln und } \\
\text { Plaques, Erosionen, Vesikel, } \\
\text { keine Schleimhautbeteiligung, } \\
\text { starker Pruritus }\end{array}$ & $\begin{array}{l}\text { 1/160 - } 200 \text { Schwangerschaften, } \\
\text { gesunde Neugeborene, unauffälliger } \\
\text { Schwangerschaftsverlauf, spontane } \\
\text { Rückbildung nach der Geburt, selten } \\
\text { Rezidive bei weiteren } \\
\text { Schwangerschaften }\end{array}$ & $\begin{array}{l}\text { lokal: } \\
\text { Kortikosteroide, antipruriginöse } \\
\text { und pflegende Externa } \\
\text { intern: } \\
\text { Antihistaminika oder/und } \\
\text { Kortikosteroide }\end{array}$ \\
\hline 2. Prurigo gestationis & 2.-3. Trimenon & $\begin{array}{l}\text { Seropapeln, sehr starker } \\
\text { Juckreiz }\end{array}$ & $\begin{array}{l}\text { gesunde Neugeborene, kein Rezidiv } \\
\text { bei erneuter Schwangerschaft }\end{array}$ & $\begin{array}{l}\text { lokal: } \\
\text { antipruriginöse und pflegende, } \\
\text { evtl. auch kortikosteroid- } \\
\text { haltige Externa }\end{array}$ \\
\hline $\begin{array}{l}\text { 3. Papulöse Dermatitis } \\
\text { in der Schwangerschaft }\end{array}$ & 1.-3. Trimenon & $\begin{array}{l}\text { (Sero-)Papeln mit zentraler } \\
\text { hämorrhagischer Kruste am } \\
\text { gesamten Integument, rasche } \\
\text { Rückbildung unter Hyper- } \\
\text { pigmentierung, starker } \\
\text { Juckreiz }\end{array}$ & $\begin{array}{l}\text { 1/2500 Schwangerschaften, } 27 \% \\
\text { Aborte und Totgeburten, vermehrt } \\
\text { Frühgeburten, erhöhte Chorion- } \\
\text { gonadotropinspiegel im Urin, rasche } \\
\text { Abheilung nach der Entbindung }\end{array}$ & $\begin{array}{l}\text { lokal: } \\
\text { pflegend, antipruriginös } \\
\text { intern: } \\
\text { Kortikosteroide }\end{array}$ \\
\hline 4. Herpes gestationis & $\begin{array}{l}\text { 2. }-3 \text {. Trimenon, } \\
\text { selten postpartal }\end{array}$ & $\begin{array}{l}\text { Polymorph: Blasen, elevierte } \\
\text { oder urtikarielle Erytheme } \\
\text { (Bild ähnlich bullösem Pemphi- } \\
\text { goid), Schleimhautbeteiligung } \\
\text { bei } 20 \% \text {, intensiver Juckreiz }\end{array}$ & $\begin{array}{l}\text { 1/50000 Schwangerschaften, 15-30\% } \\
\text { Frühgeburten und reduziertes Geburts- } \\
\text { gewicht, 5-10\% der Neugeborenen } \\
\text { weisen milde und selbstlimitierende } \\
\text { Hautveränderungen i. S. eines Herpes } \\
\text { gestationis auf. Rückbildung nach } \\
\text { Entbindung, Rezidiv in der nächsten } \\
\text { Schwangerschaft, unter Hormongabe, } \\
\text { bei Blasenmole und Chorionkarzinom } \\
\text { möglich, } \\
\text { IIF: Anti-BMZ-Antikörper („HG-Faktor“) } \\
\text { gegen BPAG } 2 \text { im Serum } \\
\text { DIF: lineäre C3-Niederschläge entlang } \\
\text { BMZ in } 100 \%, \text { IgG in } 40 \%\end{array}$ & $\begin{array}{l}\text { lokal: } \\
\text { Kortikosteroide, } \\
\text { antipruriginöse Externa } \\
\text { intern: } \\
\text { Kortikosteroide }\end{array}$ \\
\hline $\begin{array}{l}\text { 5. Autoimmun- } \\
\text { Progesteron- } \\
\text { Dermatitis }\end{array}$ & 1. Trimenon & $\begin{array}{l}\text { akneähnliche Effloreszenzen } \\
\text { an Gesäß und Extremitäten, } \\
\text { kaum Juckreiz, Arthralgien }\end{array}$ & $\begin{array}{l}\text { äußerst selten, Überempfindlichkeit } \\
\text { gegenüber endogenem Progesteron, } \\
\text { positive Hautteste und LTT mit } \\
\text { Progesteron, Eosinophilie, BSG- } \\
\text { Beschleunigung und Erhöhung von IgM } \\
\text { und IgG im Serum, meist Spontanaborte, } \\
\text { dann rasche Abheilung, Rezidiv in jeder } \\
\text { neuen Schwangerschaft oder unter oraler } \\
\text { Kontrazeption }\end{array}$ & $\begin{array}{l}\text { keine Therapie bekannt, } \\
\text { symptomatische Therapie der } \\
\text { Hauterscheinungen }\end{array}$ \\
\hline $\begin{array}{l}\text { 6. Pruritus gravidarum } \\
\text { (Synonym: benigne } \\
\text { intrahepatische Cholestase } \\
\text { bei Schwangerschaft) }\end{array}$ & 3. Trimenon & $\begin{array}{l}\text { generalisierter Pruritus sine } \\
\text { materia, eventuell Exkorationen }\end{array}$ & $\begin{array}{l}\text { Nausea, Vomitus, Anorexie, Krankheits- } \\
\text { gefühl, Dunkelfärbung des Urins, heller } \\
\text { Stuhl, Ikterus, Hepatomegalie, häufig } \\
\text { Frühgeburten, Rezidive bei weiteren } \\
\text { Schwangerschaften möglich }\end{array}$ & $\begin{array}{l}\text { lokal: } \\
\text { antipruriginöse, zinkhaltige } \\
\text { und pflegende Externa } \\
\text { intern: } \\
\text { Antihistaminika, } \\
\text { Cholestyramin }\end{array}$ \\
\hline $\begin{array}{l}\text { 7. Erythema nodosum } \\
\text { gravidarum }\end{array}$ & 1.-(2.) Trimenon & $\begin{array}{l}\text { nodöse Erytheme an typischer } \\
\text { Lokalisation, schmerzhaft }\end{array}$ & $\begin{array}{l}\text { selten, Rezidive möglich, Rückbildung } \\
\text { spontan noch während der Schwanger- } \\
\text { schaft }\end{array}$ & $\begin{array}{l}\text { lokal: } \\
\text { kortikosteroidhaltige Externa, } \\
\text { Kompressionsverbände und } \\
\text { Hochlagerung der Beine }\end{array}$ \\
\hline 8. pruritische Follikulitis & 2.-3. Trimenon & follikulär juckende Papeln & $\begin{array}{l}\text { intensiver Juckreiz, keine Rezidive } \\
\text { bei weiteren Schwangerschaften }\end{array}$ & lokal: antipruriginöse Externa \\
\hline
\end{tabular}

Die Abgrenzung zu anderen Schwangerschaftsdermatosen ist hinsichtlich ihrer Therapie und Prognose von großer praktischer Bedeutung (Tab.1). Bei Vorliegen einer PEP sind weder für die Mutter noch für das Kind bleibende Schädigungen und nur selten Rezidive in weiteren Schwangerschaften $\mathrm{zu}$ erwarten $[3,5,7,14,18]$.

Die Häufigkeit der PEP wird mit 1 von 160 [5] bis 1 von 200 [7] aller Schwangerschaften angegeben. Die meisten Publikationen bestätigen das Auftreten der Hautveränderungen im zweiten oder häufiger im letzten Drittel der Schwangerschaft $[1,3,5,8,10,14,17,18]$. In den von uns beobachteten Fällen trat die Erkrankung im sechsten Schwangerschaftsmonat erstmals auf.

Primär befallen sind nach Literaturangaben meist das Abdomen, hier insbesondere innerhalb der Striae gravidarum. Für eine unserer zwei Patientinnen konnten wir dies nicht bestätigen, da zuerst die Haut an Armen und Beinen betroffen war.

PEP tritt sowohl bei Erstgraviden, wie auch bei Zweit- und Drittschwangerschaften auf $[1,3,5,10,16,17]$. In unseren beiden Fällen waren Erstgebärende betroffen. Übereinstimmend wird bei allen Autoren eine geringe Rezidivneigung von PEP bei Folgeschwan- 
gerschaften und von PEP-ähnlichen Hauterscheinungen bei Einnahme von oralen Kontrazeptiva und Menstruation angegeben.

Die Diagnose PEP wird aus der Anamnese und dem klinischen Bild gestellt. Histologie und negative Immunhistologie sichern die klinische Diagnose. Histologisch werden zwei verschiedene Subtypen der PEP beschrieben. Die erste Form geht mit dermalen (perivaskuläre lymphohistiozytäre Infiltrate und Ödem) und epidermalen Veränderungen (Spongiose, Akanthose und Parakeratose) einher. Die zweite Form weist lediglich die beschriebenen dermalen Veränderungen auf $[4,10]$. Bei unseren beiden Patientinnen konnten histologisch sowohl dermale als auch epidermale Veränderungen gesichert werden.

In nahezu allen Kasuistiken fanden sich negative DIF-Befunde $[5,6,8-10,17]$. Ausnahmen hiervon stellen die von Alcalay et al. beschriebenen 5 Fälle mit minimalen C3-Niederschlägen an der dermo-epidermalen Junktionszone dar, denen die Autoren aber keine diagnostische Bedeutung beimessen [1] und die Beschreibung von drei positiven DIF-Befunden (einmal Fibrin perivaskulär und zweimal C3 in den dermalen Gefäßwänden) durch Yancey et al. ohne diagnostische Relevanz [18]. Vaughan Jones fand bei 44 beobachteten PEP-Fällen nur 3 mit einer gesprenkelten IgM-Ablagerung und 2 mit diskreten C3-Ablagerungen entlang der BMZ [16]. Die indirekte Immunfluoreszenz war in allen Fällen negativ. Die bei unserer Patientin 2 nachgewiesenen Antikörper gegen Interzellularsubstanz (ICS) ließen uns anfangs auch an das Vorliegen eines Pemphigus vulgaris denken. Es konnte im Rahmen der Diagnostik jedoch gezeigt werden, dass die Bindung der Antikörper an die ICS nach Absorption mit den Blutgruppensubstanzen A und B ausblieb. Es handelt sich also bei diesen so genannten Pemphigus-like-Antikörpern um Blutgruppenisoagglutinine, die nach Absorption keine netzförmige Fluoreszenz auf dem Substrat mehr zeigten. Pemphigus-like-Antikörper können nach Verbrennungen, Medikamenteneinnahme (z. B. Penicillamin) oder als Blutgruppenisoagglutinine auftreten [13]. Bei der zweiten Patientin fielen im Rahmen der Diagnostik positive Masern-Antikörpertiter auf, die für eine frische Maserninfektion sprechen könnten. Das klinische Bild entsprach jedoch nicht dieser Verdachtsdiagnose. Eine geplante Titerverlaufskontrolle konnte nicht erfolgen, da die Patientin die Klinik verlassen hatte.

Ätiologie und Pathogenese der PEP sind bis heute unklar. Ältere Arbeiten diskutierten als möglichen Auslöser die Dehnung der abdominellen Haut, was jedoch die Hautveränderungen im Bereich anderer Körperstellen nicht erklärt $[1,3,5-8,10,16,17]$. Auch eine familiäre Veranlagung wurden diskutiert [17]. Ebenfalls wurde die Einnahme bestimmter Medikamente für das Auftreten von PEP angeschuldigt, u.a. Multivitaminpräparate in Überdosen, Eisenpräparate und Laxantien [14].

Saraswat et al. beschrieben den interessanten Fall einer 53jährigen Frau mit PEP-ähnlichen Hauterscheinungen bei Vorliegen eines Zervixkarzinoms [15]. Sie gab juckende Hauterscheinungen in allen vier Schwangerschaften an, die letzte lag 28 Jahre zurück. Diskutiert wird hier die ektope Produktion von humanem Choriongonadotropin durch den Tumor als mögliche Ursache für diesen Fall einer PEP-ähnlichen Dermatose.
Tab. 2 Systemische Steroidtherapie in der Schwangerschaft [11]

strenge Indikationsstellung
so kurz wie möglich
so niedrig wie möglich
Langzeitdosis möglichst unter $10 \mathrm{mg}$ Prednisolonäquivalent
1. Trimenon: Methylprednisolon > Prednisolon
2. und 3. Trimenon: Methylprednisolon oder Prednisolon
Dexamethason oder Betamethason nur bei kindlicher Indikation, nicht in der
Stillzeit

Therapeutisch wurden in fast allen Kasuistiken milde Anwendungen von topischen Steroiden und antipruriginösen Externa als ausreichend beschrieben, gegebenenfalls zusätzlich in einigen Fällen kombiniert mit interner Antihistaminikagabe. Auch interne niedrigdosierte Steroidmedikationen waren in einigen Fällen bei ausgedehnten Lokalbefunden und stärkstem Juckreiz notwendig $[6,7,10,13,16,18]$. Pfeiffer hat für eine systemische Steroidtherapie in der Schwangerschaft folgende Empfehlungen veröffentlicht (Tab. 2) [11]. Bei unseren Patientinnen entschieden wir uns aufgrund der Ausdehnung der PEP und des geklagten Pruritus für eine kurzzeitige niedrigdosierte interne Gabe von Prednisolon. Patientin 1 hatte im Vorfeld von einer externen Therapie in Kombination mit einer internen Antihistaminikagabe nur kurzzeitig profitiert, es kam rasch zu einem Rezidiv. Bereits nach wenigen Tagen der Prednisolontherapie kam es bei beiden Patientinnen zu einer deutlichen und raschen Besserung von Hauterscheinungen und Juckreiz.

Differenzialdiagnostisch sollten andere Schwangerschaftsdermatosen und schwangerschaftsunabhängig auftretende Dermatosen abgeklärt werden (Tab.1).

\section{Literatur}

${ }^{1}$ Alcalay J, Ingber A, David M, Hazaz B, Sandbank M. Pruritic Urticarial Papules and Plaques of Pregnancy. A Review of 21 cases. J Reprod Med 1987; 32: $315-316$

2 Al Fares SI. The specific dermatoses of pregnancy: a re-appraisal. J Eur Acad Dermatol Venereol 2001; 15: 197-206

${ }^{3}$ Aronson IK, Bond S, Fiedler VC, Vomvouras S, Gruber D, Ruiz C. Pruritic urticarial papules and plaques of pregnancy: Clinical and immunopathologic observations in 57 patients. J Am Acad Dermatol 1998; 39: 933-939

${ }^{4}$ Baum EW, Wade TR. Pathology of PUPPP. J Am Acad Dermatol 1982; 6 : $402-403$

${ }^{5}$ Charles-Holmes R. Polymorphic Eruption of Pregnancy. Semin Dermatol 1989; 8: $18-22$

${ }^{6}$ Cohen LM, Capeless EL, Krusinski PA, Maloney ME. Pruritic Urticarial Papules and Plaques of Pregnancy and Its Relationship to Maternal-Fetal Weight Gain and Twin Pregnancy. Arch Dermatol 1989; 125: $1534-1536$

${ }^{7}$ Elling SV, Mc Kenna P, Powell FC. Pruritic urticarial papules and plaques of pregnancy in twin and triplet pregnancies. J Eur Acad Dermatol Venereol 2000; 14: 378-381

${ }^{8}$ Fox GN. Pruritic Urticarial Papules and Plaques of Pregnancy. Am Fam Physician 1986; 34: 191 - 195

${ }^{9}$ Kasp-Grochowska E, Beck J, Holmes RC, Black MM, Timlin D. The Role of Circulating Immune Complexes in the Aetiology of Polymorphic Eruption of Pregnancy. Arch Dermatol Res 1984; 276: 71 - 73 
${ }^{10}$ Lawley TJ, Hertz KC, Wade TR, Ackermann AB, Katz SI. Pruritic Urticarial Papules and Plaques of Pregnancy. JAMA 1979; 241: 1696-1699

${ }^{11}$ Pfeiffer C. Dermatologische Steroidtherapie in der Schwangerschaft und Stillzeit. Hautarzt 2001; 52: $998-1002$

12 Rahbari H. Priority for PUPPP. J Am Acad Dermatol 1982; 6: 947-948

${ }^{13}$ Rasmussen HB, Jepsen LV, Brandrup F. Penicillamine induced bullous pemphigoid with pemphigus-like antibodies. J Cutan Pathol 1989; 16: $154-157$

${ }^{14}$ Röckl H, Lurz C. Die PUPPP-Dermatose (Pruritic Urticarial Papules and Plaques of Pregnancy). Hautarzt 1983; 34: 179-181

15 Saraswat A, Rai R, Kumar B. Lesions Resembling Polymorphic Eruption of Pregnancy Several Years after Pregnancy. Dermatology 2001; 202: 82

${ }^{16}$ Vaughan JonesSA, Hern S, Nelson-Piercy C, Seed PT, Black MM. A prospective study of 200 women with dermatoses of pregnancy correlating clinical findings with hormonal and immunopathological profiles. Br J Dermatol 1999; 141: 71 - 81

${ }^{17}$ Weiss R, Hull P. Familial occurrence of pruritic urticarial papules and plaques of pregnancy. J Am Acad Dermatol 1992; 26: 715-717

18 Yancey KB, Hall RP, Lawley TJ. Pruritic urticarial papules and plaques of pregnancy. Clinical experience in twenty-five patients. J Am Acad Dermatol 1984; 10: $473-480$

\section{Buchbesprechung}

\section{Autoimmune Krankheiten und ihre Behandlung}

M. Sticherling, E. Christophers (eds.)

Treatment of Autoimmune Disorders. Wien: Springer 2003. 176 S., 32 Abb. Geb. 48,- . ISBN 3-211-83772-8

Bis zu 5\% der Erwachsenen leiden unter Autoimmunkrankheiten oder zeigen Autoimmunphänomene. Das Risiko ist durch Schwangerschaften erhöht und steigt im Alter. Besonders betroffene Organe sind das Nervensystem, die Augen, der Magendarmtrakt und die Haut. Organspezifische Erfahrungen gehören ausgetauscht und gebündelt. Reichlich Erfahrung wurde durch die Organtransplantation gewonnen, im Spektrum der Manifestationen und ganz besonders in der Behandlung und deren Kontrolle. Dies zusammenfassend, bemüht sich die „German Society of Autoimmune Diseases“ mit jährlichen, internationalen Symposien um Fortschritt und Verständnis.

Im vorgelegten Band sind 15 Artikel enthalten, von Spezialisten für Fachleute, mit einer Fülle von Daten, Methoden und Resultaten und mit ausführlicher Literaturdokumentation. Arbeitsgruppen aus Deutschland, USA, Kanada, Australien, Israel, England, Frankreich, Belgien und der Schweiz sind beteiligt.

Schwerpunkte beleuchten die Coeliakie, die Multiple Sklerose, die rheumatische Arthritis (besonders in der Schwangerschaft), blasenbildende Hautkrankheiten und die Uveitis. Therapeutisch werden die antigenspezifischen und die unspezifischen Immunsuppressionen, die adjuvanten Schemata, Toleranzinduktion, Vakzinationen und intravenöse Gaben von Immunglobulinen untersucht und kritisch bearbeitet. Ein Buch, das den Fortschritt beschreibt und enorm fördert. 